\title{
Distribuição dos valores do índice de massa corporal da população brasileira até 25 anos
}

\author{
Luiz Antonio dos Anjos, ${ }^{1}$ Gloria Valeria da Veiga ${ }^{2}$ \\ e Inês Rugani Ribeiro de Castro ${ }^{3}$
}

RESUMO O presente artigo apresenta a distribuição em percentis do indice de massa corporal da população jovem brasileira. As medidas de massa corporal e estatura de crianças e jovens de ambos os sexos, do nascimento até os 25 anos, foram obtidas a partir da Pesquisa Nacional sobre Saúde e Nutrição realizada pelo Instituto Brasileiro de Geografia e Estatística e pelo Instituto Nacional de Alimentação e Nutrição em 1989, com o objetivo de descrever o estado de saúde e nutrição da população brasileira. Os valores do indice de massa corporal levantados pela pesquisa tendem a decrescer na faixa etária de 1 a 6 anos, mantêm-se estáveis até cerca de 8 anos e aumentam progressivamente, até se estabilizarem por volta de 19 a 20 anos para mulheres e 20 a 21 anos para homens. A pesquisa também revelou que as meninas apresentam valores de indice de massa corporal superiores aos dos meninos a partir dos 12 anos, bem como maiores diferenças entre valores de percentis extremos (percentil 3 e 97). A comparação com dados de outros países revelou que, em linhas gerais, o padrão de evolução do indice de massa corporal no Brasil assemelha-se ao encontrado na França, Grã-Bretanha e Estados Unidos. As crianças e jovens brasileiros apresentam valores médios de indice de massa corporal semelhantes aos norteamericanos até os 6 anos e inferiores a partir dos 7 anos. A comparação entre a evolução da mediana do indice de massa corporal em crianças e adolescentes brasileiros e britânicos revelou valores sempre inferiores para as meninas brasileiras. Em relação à França, o índice de massa corporal da população masculina brasileira é sistematicamente menor a partir dos 8 anos. Os dados aqui apresentados podem servir para acompanhamento de tendências e comparações entre estudos; seu uso para fins de triagem e monitoramento clínico é desaconselhável, dada a ampla variação individual no processo de crescimento e desenvolvimento durante a adolescência.

1 Fundação Oswaldo Cruz, Escola Nacional de Saúde Pública, e Universidade Federal Fluminense, Faculdade de Nutrição, Departamento de Nutrição Social. Correspondência e pedidos de separatas devem ser enviados para este autor no seguinte endereço: Escola Nacional de Saúde Pública, Fundação Oswaldo Cruz, Rua Leopoldo Bulhões, 1480, Manguinhos, CEP 21041-210, Rio de Janeiro, RJ, Brasil. Correio eletrônico: anjos@manguinhos.ensp. fiocruz.br

2 Universidade Federal Fluminense, Faculdade de Nutrição, Departamento de Nutrição e Dietética, RJ, Brasil. Atualmente na Universidade Federal do Rio de Janeiro, Instituto de Nutrição, Departamento de Nutrição Social e Aplicada, RJ, Brasil.

3 Universidade do Estado do Rio de Janeiro, Instituto de Nutrição, Departamento de Nutrição Social e Aplicada e Secretaria Municipal de Saúde do Rio de Janeiro, RJ, Brasil. , RJ, Brasil.
A adolescência é uma fase caracterizada por alterações morfológicas, fisiológicas, psicológicas e sociais intensas e complexas, nas quais a nutrição desempenha um importante papel (1). É durante a adolescência, por exemplo, que o indivíduo adquire aproximadamente $25 \%$ de sua estatura final e $50 \%$ de sua massa corporal (2). Alterações importantes também ocorrem na composição corporal, caracterizadas por depósito maior de gordura em meninas e de massa muscular em meninos $(3,4)$.

Além da rapidez com que estas mudanças ocorrem, na adolescência os fa- tores genéticos e étnicos passam a ser os principais fatores de influência sobre o desenvolvimento, contrastando com a infância, em que se destacam os fatores ambientais. Como resultado, existem enormes variações entre indivíduos e populações em relação ao início, duração, seqüência e magnitude dos eventos pubertários, principalmente entre 10 e 14 anos (3). A idade cronológica, portanto, perde parte de sua importância como condicionante do crescimento e desenvolvimento e dá lugar à maturação sexual como principal variável, traduzida pelo desen- 
volvimento das características sexuais secundárias $(5,6)$. Adolescentes da mesma idade e gênero, com a mesma massa corporal e estatura, podem atravessar fases idênticas de seu desenvolvimento em momentos diferentes. Dessa forma, os critérios antropométricos para avaliação nutricional na adolescência são mais complexos do que os critérios para avaliar crianças menores de 10 anos, sobre os quais existe consenso (7-9).

A avaliação antropométrica do estado nutricional de adolescentes em estudos populacionais ainda é tema de grande polêmica na literatura. Concorda-se, porém, que é fundamental a avaliação da composição corporal (10) com incorporação do estagiamento maturacional (3). Com relação à avaliação antropométrica, tem-se sugerido desde adaptações de curvas americanas da relação massa corporal para estatura (11) até a utilização de pontos de corte a partir da distribuição do índice de massa corporal (IMC) na população (12).

Em países em desenvolvimento, além do diagnóstico de obesidade, persiste o desafio do diagnóstico de deficiência energética que leva a quadros de desnutrição (13). No caso destes países, não é recomendável o uso de dados de massa corporal e estatura - nem de índices destas medidas, como o índice de massa corporal obtidos a partir de estudos feitos em países desenvolvidos. Isto se aplica particularmente aos adolescentes, devido à grande variação na estatura atingida durante esta fase, influenciada por fatores genéticos e ambientais (14). Em países em desenvolvimento, grandes proporções de jovens apresentam baixa estatura, muitas vezes como conseqüência de agravos nutricionais na infância (15). Portanto, utilizar como parâmetro os valores de IMC obtidos de populações cujos jovens alcançam seu potencial genético de crescimento pode levar a erros na avaliação nutricional. É necessário desenvolver uma metodologia antropométrica que avalie a relação entre massa corporal e estatura e que leve em consideração tanto um possível déficit estatural quanto o momento da maturação sexual em que se encontra o adolescente. Entretanto, são escassos os estudos populacionais sobre composição corporal associada à maturação sexual $(16,17)$.

O objetivo do presente trabalho é apresentar a distribuição do IMC da população brasileira desde o nascimento até os 25 anos. Nosso intuito é contribuir para a discussão sobre avaliação nutricional dos adolescentes brasileiros, na perspectiva de auxiliar na construção de curvas nacionais que possam ser utilizadas em estudos populacionais para acompanhamento de tendências e comparação com outros países.

\section{MATERIAIS E MÉTODOS}

O presente estudo é do tipo descritivo. O trabalho apresenta a distribuição dos valores de IMC da população brasileira com base nos resultados da Pesquisa Nacional sobre Saúde e Nutrição (PNSN), realizada pelo Instituto Brasileiro de Geografia e Estatística (IBGE) e pelo Instituto Nacional de Alimentação e Nutrição (INAN) em 1989. Tratou-se de um levantamento nacional com amostra probabilística da população brasileira, cujo objetivo central foi descrever o estado de saúde e nutrição da população no seu contexto bio-socioeconômico. Para tanto, utilizou-se uma amostragem complexa, de base domiciliar, cujos âmbitos de análise foram as cinco grandes regiões geográficas brasileiras (Norte, Nordeste, Sudeste, Sul e Centro-Oeste) e as situações urbana e rural de cada região, com exceção do Norte rural, por problemas operacionais. Portanto, nove âmbitos foram analisados (18).

$\mathrm{Na}$ primeira etapa da amostragem da PNSN, os nove âmbitos da pesquisa foram estratificados em três níveis socioeconômicos através de informações de renda domiciliar média do setor demográfico e da taxa de alfabetização da população de 5 a 15 anos, baseadas em projeções dos dados do censo nacional de 1980. Para cada estrato foram sorteados 18 setores censitários, totalizando 54 setores em cada âmbito e 486 em todo o país. Em uma segunda fase, 36 domicílios (em média) foram sorteados para cada setor, totalizando 14455 domicílios investigados, com dados sobre 63213 brasileiros de todas as idades (19).

A casuística do presente trabalho compreendeu todos os indivíduos com até 25 anos de idade, não portadores de deficiência física que prejudicasse a avaliação de estatura e massa corporal. As mulheres grávidas foram eliminadas da análise. No total, dados de 16641 e 17135 pessoas do sexo feminino e masculino, respectivamente, foram analisados.

Além das medidas antropométricas de massa corporal e estatura, núcleo central da PNSN, foram coletadas, em questionários padronizados, informações sobre características demográficas básicas e de saúde. Todas as informações foram coletadas nos domicílios por duplas de entrevistadores treinados (antropometrista e anotador). O IMC foi calculado pela divisão do valor da massa corporal em quilogramas pelo quadrado da estatura em metros $\left(\mathrm{IMC}=\mathrm{kg} / \mathrm{m}^{2}\right)$. O IMC é utilizado largamente na avaliação do estado nutricional de adultos por vários motivos: 1) sua alta correlação com a massa corporal e indicadores de composição corporal; 2) sua capacidade de predizer riscos de patologias (20); e 3) a não necessidade de se usar dados de referência antropométricos no diagnóstico do estado nutricional, isto é, utiliza-se um ponto de corte fixo ao invés de um valor de distribuição populacional (por exemplo, um indivíduo com IMC > 30 apresenta um determinado risco relacionado à obesidade; não é necessário procurar em tabelas de distribuição de massa corporal o valor de massa "ideal" para a sua estatura e em que proporção este valor estaria acima de um valor teórico "ideal") (13). No presente trabalho, percentis selecionados de IMC foram calculados, por sexo e faixa etária. Os percentis selecionados foram os tradicionalmente utilizados em estudos de distribuição de valores antropométricos populacionais.

O programa estatístico SAS (21) foi utilizado para o cálculo dos percentis não suavizados e dos valores mé- 
TABELA 1. Distribuição dos valores do índice de massa corporal $\left(\mathrm{kg} / \mathrm{m}^{2}\right)$ da população feminina brasileira em função da faixa etária, Brasil, 1989a

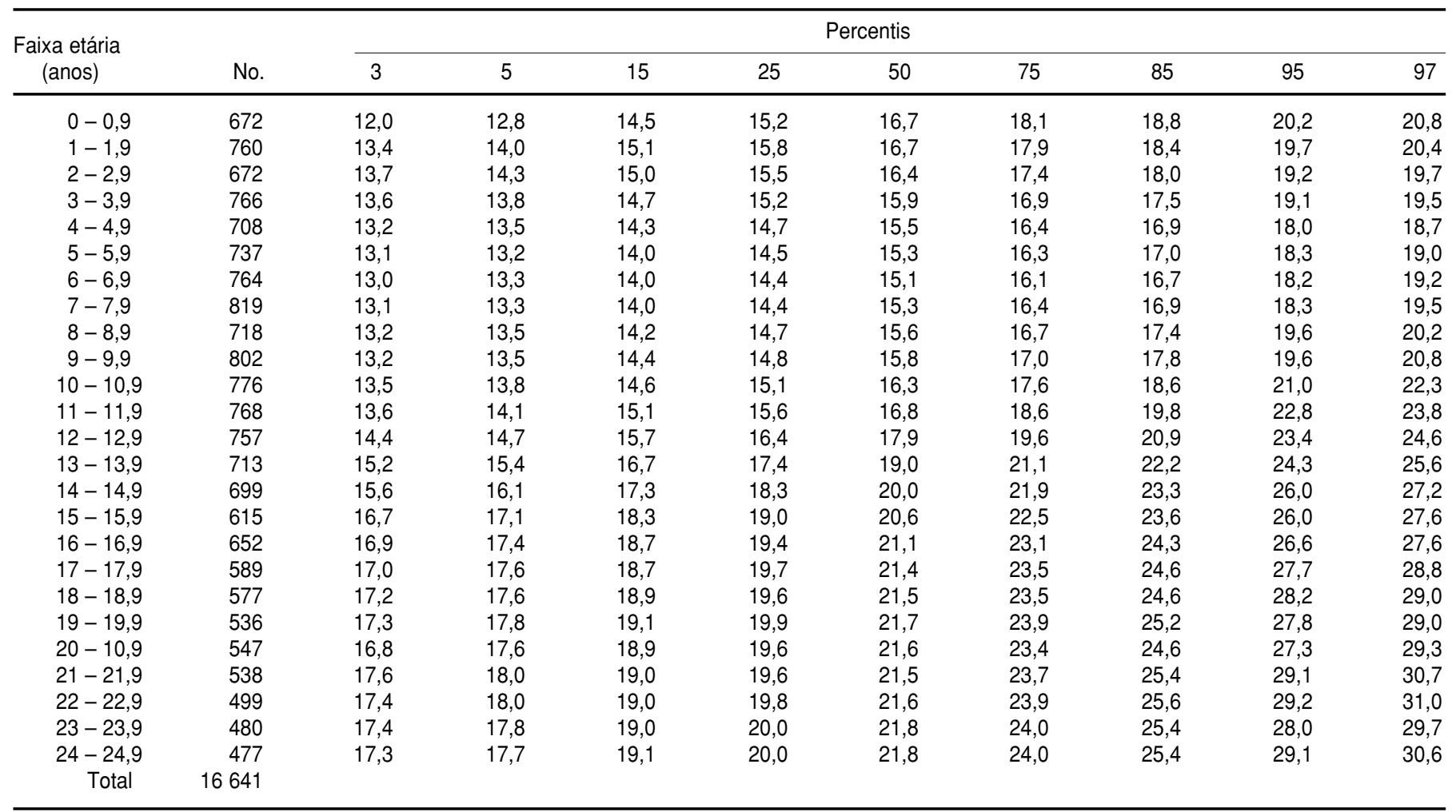

a Instituto Brasileiro de Geografia e Estatística e Instituto Nacional de Alimentação e Nutrição, dados da Pesquisa Nacional sobre Saúde e Nutrição, 1989.

dios por sexo e faixa etária. A opção WEIGHT foi utilizada de forma a incluir o fator de expansão da amostra, calculada como inverso da probabilidade de seleção dos elementos (domicílios ou pessoas).

\section{RESULTADOS}

A distribuição dos valores de IMC de crianças e jovens brasileiros de ambos os sexos, de 0 a 25 anos, obtidos na PNSN, está apresentada nas tabelas 1 e 2 e ilustrada, para percentis selecionados, nas figuras 1 e 2 . Observa-se que os valores de IMC decrescem de 1 a 6 anos, mantêm-se estáveis até cerca dos 8 anos, voltam a aumentar até o fim da faixa etária estudada e se estabilizam em torno dos 19 ou 20 anos para mulheres e dos 20 ou 21 anos para homens.

Até os 11 anos, aproximadamente, os valores medianos de IMC são se- melhantes entre meninos e meninas; até os 8 anos estes valores são ligeiramente inferiores para meninas. Dos 10 aos 19,9 anos, as meninas sistematicamente apresentam valores mais altos. A diferença entre os valores medianos (percentil 50), que é de 0,2 unidades de IMC (UIMC) no 11. ano de vida, alcança 1,7 UIMC na faixa de 14 a 14,9 anos. Esta diferença decresce no período subseqüente e chega a 0,5 UIMC na faixa de 19 a 19,9 anos.

A comparação entre os valores dos percentis (P) extremos (3 e 97), para ambos os sexos (tabela 3 ), revela que, até os 10 anos, a diferença entre o $\mathrm{P} 97$ e o P 3 alcança, no máximo, 9,2 UIMC. A partir de então, as meninas apresentam diferenças sempre maiores do que os meninos. Para meninos, a menor e a maior diferenças ocorrem no $11^{\circ}$ ano de vida (7,5 UIMC) e no $25^{\circ}$ ano (12 UIMC), respectivamente; enquanto que, para meninas, a menor e a maior diferenças ocorrem no 11. (8,8 UIMC) e 23. ano (13,6 UIMC), respectivamente. Esta maior amplitude nos valores de IMC para o sexo feminino pode ser visualizada pela comparação das figuras 1 e 2 .

\section{DISCUSSÃO E CONCLUSÕES}

$\mathrm{O}$ aumento dos valores de IMC ao longo da adolescência pode ser interpretado como um marcador de amadurecimento orgânico que expressa aumento na proporção tronco/membros, na massa corporal, na estatura e nas alterações da composição corporal. As diferenças sexuais no ritmo de amadurecimento, como a precocidade feminina em relação aos estagiamentos maturacionais $(22,23)$, explicam o padrão encontrado nas curvas brasileiras, onde os valores medianos para meninas, que são sistematicamente superiores aos valores encontrados para meninos entre 12 e 20 anos, apresen- 
TABELA 2. Distribuição dos valores do índice de massa corporal $\left(\mathrm{kg} / \mathrm{m}^{2}\right)$ da população masculina brasileira em função da faixa etária, Brasil, 1989a

\begin{tabular}{|c|c|c|c|c|c|c|c|c|c|c|}
\hline \multirow{2}{*}{$\begin{array}{c}\text { Faixa etária } \\
\text { (anos) }\end{array}$} & \multirow[b]{2}{*}{ No. } & \multicolumn{9}{|c|}{ Percentis } \\
\hline & & 3 & 5 & 15 & 25 & 50 & 75 & 85 & 95 & 97 \\
\hline $0-0,9$ & 685 & 12,6 & 13,0 & 14,8 & 15,7 & 17,4 & 18,7 & 19,4 & 20,9 & 21,8 \\
\hline $2-2,9$ & 701 & 14,5 & 14,7 & 15,5 & 15,8 & 16,7 & 17,7 & 18,2 & 19,3 & 19,9 \\
\hline $3-3,9$ & 776 & 13,6 & 14,2 & 15,0 & 15,4 & 16,2 & 17,1 & 17,6 & 18,6 & 19,2 \\
\hline $4-4,9$ & 754 & 13,5 & 13,9 & 14,6 & 15,1 & 15,8 & 16,7 & 17,2 & 18,4 & 18,7 \\
\hline $7-7,9$ & 879 & 13,4 & 13,5 & 14,2 & 14,6 & 15,5 & 16,3 & 16,9 & 18,6 & 20,0 \\
\hline $8-8,9$ & 803 & 13,4 & 13,7 & 14,3 & 14,8 & 15,6 & 16,6 & 17,2 & 18,5 & 20,0 \\
\hline $9-9,9$ & 767 & 13,4 & 13,7 & 14,5 & 15,0 & 15,9 & 16,9 & 17,5 & 19,6 & 21,2 \\
\hline $10-10,9$ & 780 & 13,7 & 14,1 & 14,8 & 15,2 & 16,1 & 17,1 & 18,0 & 20,0 & 21,2 \\
\hline $11-11,9$ & 782 & 13,9 & 14,3 & 15,1 & 15,6 & 16,5 & 17,6 & 18,7 & 21,3 & 22,4 \\
\hline $12-12,9$ & 721 & 14,1 & 14,5 & 15,4 & 15,9 & 16,9 & 18,2 & 19,1 & 22,0 & 23,1 \\
\hline $13-13,9$ & 732 & 14,8 & 15,1 & 16,0 & 16,5 & 17,7 & 19,2 & 20,1 & 21,9 & 23,4 \\
\hline $18-18,9$ & 624 & 17,3 & 17,8 & 19,1 & 19,6 & 21,0 & 22,4 & 23,3 & 25,1 & 25,7 \\
\hline $19-19,9$ & 542 & 17,9 & 18,1 & 19,2 & 19,8 & 21,2 & 22,9 & 23,8 & 25,7 & 27,4 \\
\hline $20-20,9$ & 478 & 17,9 & 18,4 & 19,6 & 20,3 & 21,7 & 23,1 & 24,1 & 25,8 & 26,7 \\
\hline $21-21,9$ & 578 & 18,1 & 18,5 & 19,7 & 20,5 & 21,8 & 23,2 & 24,0 & 26,5 & 27,6 \\
\hline $22-22,9$ & 541 & 18,0 & 18,4 & 19,7 & 20,5 & 21,9 & 23,6 & 24,9 & 26,9 & 27,8 \\
\hline $23-23,9$ & 477 & 18,4 & 18,8 & 19,8 & 20,6 & 22,0 & 23,7 & 24,6 & 26,9 & 27,8 \\
\hline $24-24,9$ & 502 & 18,1 & 18,6 & 19,9 & 20,6 & 22,4 & 24,2 & 25,4 & 28,5 & 30,1 \\
\hline Total & 17135 & & & & & & & & & \\
\hline
\end{tabular}

a Instituto Brasileiro de Geografia e Estatística e Instituto Nacional de Alimentação e Nutrição, dados da Pesquisa Nacional sobre Saúde e Nutrição, 1989.

FIGURA 1. Percentis (P) selecionados do índice de massa corporal $\left(\mathrm{kg} / \mathrm{m}^{2}\right)$ da população feminina brasileira em função da idade, 1989a

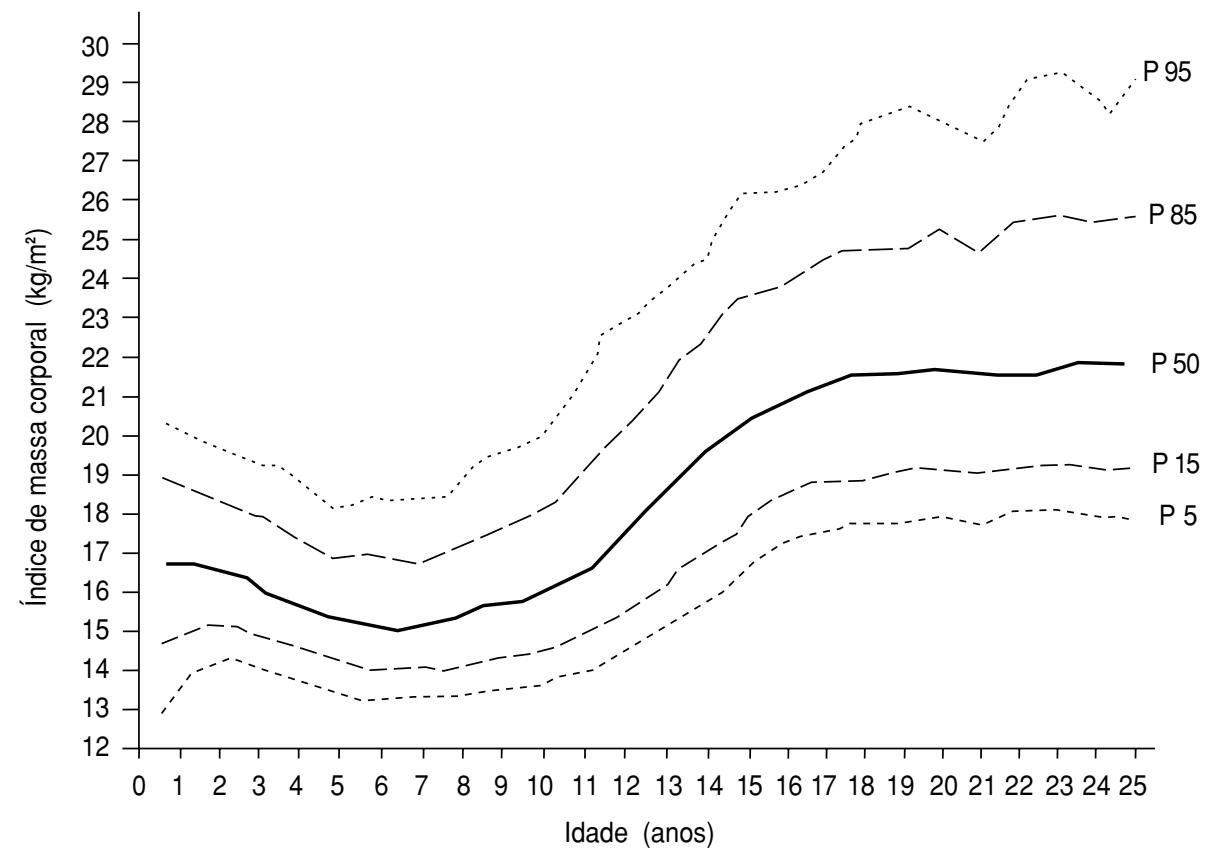

\footnotetext{
a Instituto Brasileiro de Geografia e Estatística e Instituto Nacional de Alimentação e Nutrição, dados da Pesquisa Nacional sobre Saúde e Nutrição, 1989.
} 
FIGURA 2. Percentis (P) selecionados do índice de massa corporal $\left(\mathrm{kg} / \mathrm{m}^{2}\right)$ da população masculina brasileira em função da idade, $1989^{a}$

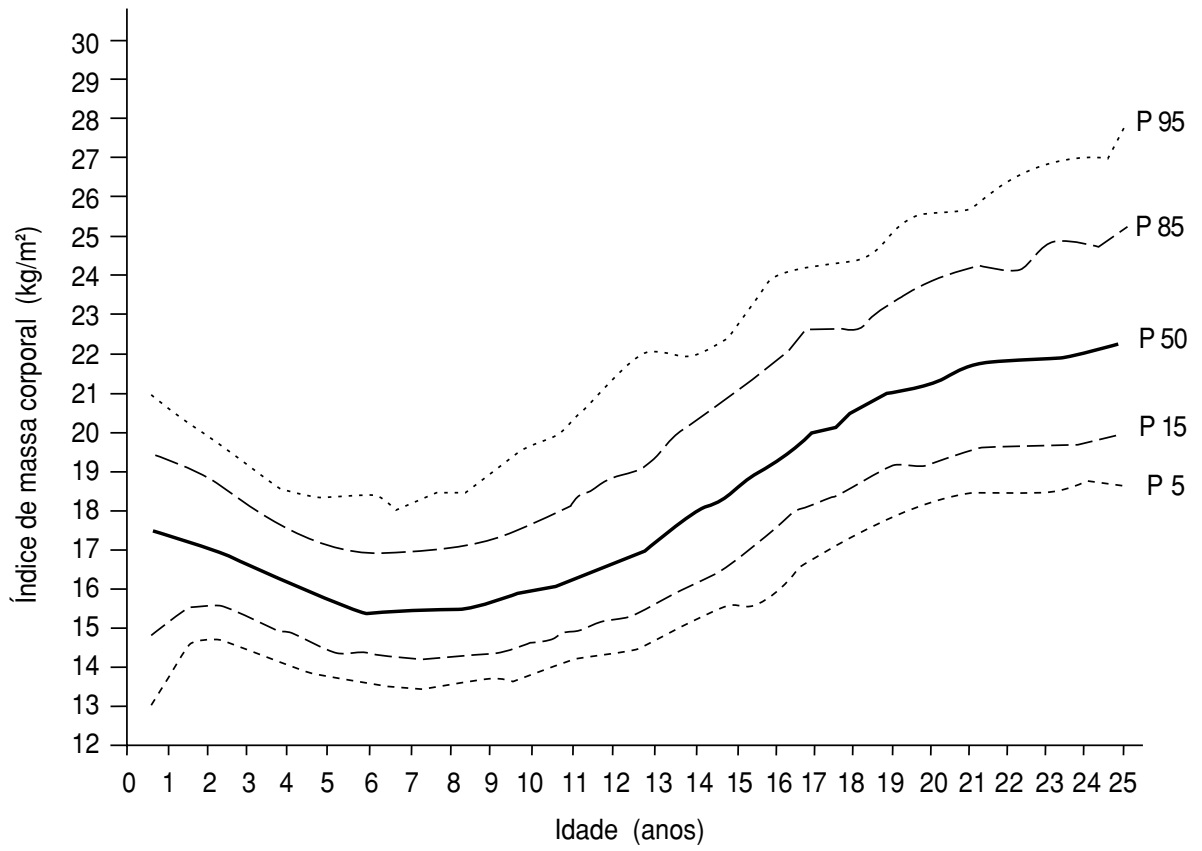

a Instituto Brasileiro de Geografia e Estatística e Instituto Nacional de Alimentação e Nutrição, dados da Pesquisa Nacional sobre Saúde e Nutrição, 1989.

TABELA 3. Valores de percentis (P) selecionados do índice de massa corporal $\left(\mathrm{kg} / \mathrm{m}^{2}\right)$ por faixa etária das populações masculina e feminina, Brasil, 1989a

\begin{tabular}{|c|c|c|c|c|c|c|c|}
\hline \multirow{2}{*}{$\begin{array}{l}\text { Faixa etária } \\
\text { (anos) }\end{array}$} & \multicolumn{3}{|c|}{ Meninas (1) } & \multicolumn{3}{|c|}{ Meninos (2) } & \multirow{2}{*}{$\frac{(1)-(2)}{a-b}$} \\
\hline & P 97 & P 3 & P $97-P 3$ (a) & P 97 & P 3 & P 97 - P 3 (b) & \\
\hline $0-0,9$ & 20,8 & 12,0 & 8,8 & 21,8 & 12,6 & 9,2 & $-0,4$ \\
\hline $1-1,9$ & 20,4 & 13,4 & 7,0 & 20,4 & 14,2 & 6,2 & 0,8 \\
\hline $2-2,9$ & 19,7 & 13,7 & 6,0 & 19,9 & 14,5 & 5,4 & 0,6 \\
\hline $3-3,9$ & 19,5 & 13,6 & 5,9 & 19,2 & 13,6 & 5,6 & 0,3 \\
\hline $4-4,9$ & 18,7 & 13,2 & 5,5 & 18,7 & 13,5 & 5,2 & 0,3 \\
\hline $5-5,9$ & 19,0 & 13,1 & 5,9 & 20,0 & 13,3 & 6,7 & $-0,8$ \\
\hline $6-6,9$ & 19,2 & 13,0 & 6,2 & 18,8 & 13,3 & 5,5 & 0,7 \\
\hline $7-7,9$ & 19,5 & 13,1 & 6,4 & 20,0 & 13,4 & 6,6 & $-0,2$ \\
\hline $8-8,9$ & 20,2 & 13,2 & 7,0 & 20,0 & 13,4 & 6,6 & 0,4 \\
\hline $9-9,9$ & 20,8 & 13,2 & 7,6 & 21,2 & 13,4 & 7,8 & $-0,2$ \\
\hline $10-10,9$ & 22,3 & 13,5 & 8,8 & 21,2 & 13,7 & 7,5 & 1,3 \\
\hline $11-11,9$ & 23,8 & 13,6 & 10,2 & 22,4 & 13,9 & 8,5 & 1,7 \\
\hline $12-12,9$ & 24,6 & 14,4 & 10,2 & 23,1 & 14,1 & 9,0 & 1,2 \\
\hline $13-13,9$ & 25,6 & 15,2 & 10,4 & 23,4 & 14,8 & 8,6 & 1,8 \\
\hline $14-14,9$ & 27,2 & 15,6 & 11,6 & 23,4 & 15,1 & 8,3 & 3,3 \\
\hline $15-15,9$ & 27,6 & 16,7 & 10,9 & 24,7 & 15,5 & 9,2 & 1,7 \\
\hline $16-16,9$ & 27,6 & 16,9 & 10,7 & 24,8 & 16,2 & 8,6 & 2,1 \\
\hline $17-17,9$ & 28,8 & 17,0 & 11,8 & 25,0 & 16,8 & 8,2 & 3,6 \\
\hline $18-18,9$ & 29,0 & 17,2 & 11,8 & 25,7 & 17,3 & 8,4 & 3,4 \\
\hline $19-19,9$ & 29,0 & 17,3 & 11,7 & 27,4 & 17,9 & 9,5 & 2,2 \\
\hline $20-20,9$ & 29,3 & 16,8 & 12,5 & 26,7 & 17,9 & 8,8 & 3,7 \\
\hline $21-21,9$ & 30,7 & 17,6 & 13,1 & 27,6 & 18,1 & 9,5 & 3,6 \\
\hline $22-22,9$ & 31,0 & 17,4 & 13,6 & 27,8 & 18,0 & 9,8 & 3,8 \\
\hline $23-23,9$ & 29,7 & 17,4 & 12,3 & 27,8 & 18,4 & 9,4 & 2,9 \\
\hline $24-24,9$ & 30,6 & 17,3 & 13,3 & 30,1 & 18,1 & 12,0 & 1,3 \\
\hline
\end{tabular}

a Instituto Brasileiro de Geografia e Estatística e Instituto Nacional de Alimentação e Nutrição, dados da Pesquisa Naciona sobre Saúde e Nutrição, 1989. tam diferenças cada vez maiores até os 15 anos - período de maior contraste entre os dois sexos - , decrescendo depois, à medida em que os estagiamentos de meninos e meninas se aproximam.

Quanto à comparação com estudos de outros países, pode-se observar que, em linhas gerais, o padrão de evolução do IMC, no Brasil, assemelha-se ao encontrado na França (24), GrãBretanha $(25,26)$ e Estados Unidos (27-30): queda dos valores entre 1 e 6 a 8 anos de idade; a partir daí, aumento constante do IMC, pelo menos até 18 anos, que é a idade passível de comparação entre quatro dos cinco estudos (os dados do estudo realizado na GrãBretanha vão somente até os 15 anos). Embora estes estudos tenham sido realizados em diferentes épocas e não apresentem metodologia idêntica (por exemplo, há diferenças no desenho - longitudinal ou transversal - e nas características amostrais) àquela utilizada em nosso estudo, eles podem ser utilizados na comparação entre os perfis globais de cada país (tabelas 4-6). Por exemplo, a comparação entre a evolução da mediana do IMC em crianças e adolescentes brasileiros e em crianças e adolescentes britânicos (tabela 4), revela que, entre meninas, os valores são sempre inferiores para a população brasileira, com diferenças que aumentam até o 12. ano de vida e decrescem depois. Em relação aos meninos, os valores passam a ser menores a partir do $6^{\circ}$ ano; as diferenças são cada vez maiores até o 10 . ano e se estabilizam em torno de 1 UIMC até o 16. ano de vida.

Já em relação ao estudo logitudinal do Fels (30) pode-se notar que, entre a população feminina, os valores médios de IMC brasileiros assemelhamse aos resultados obtidos através do primeiro modelo matemático usado no estudo norte-americano e são sempre superiores aos do segundo modelo apresentado. Entre a população masculina, os valores brasileiros são maiores do que os dois modelos aos 6 anos. Aos 12 e 18 anos os valores de IMC para os meninos brasileiros estão em situação intermediária em relação aos dois modelos. 
TABELA 4. Valores medianos do índice de massa corporal $\left(\mathrm{kg} / \mathrm{m}^{2}\right)$ das populações brasileira e britânica, distribuídos por faixa etária e sexo

\begin{tabular}{|c|c|c|c|c|}
\hline \multirow{2}{*}{$\begin{array}{l}\text { Faixa etária } \\
\left(^{(a n o s)^{c}}\right.\end{array}$} & \multicolumn{2}{|c|}{ População brasileira ${ }^{a}$} & \multicolumn{2}{|c|}{ População britânicab } \\
\hline & Meninos & Meninas & Meninos & Meninas \\
\hline $3-3.9$ & 16.2 & 15.9 & 16.2 & 16.0 \\
\hline $4-4,9$ & 15,8 & 15,5 & 15,8 & 15,7 \\
\hline $5-5,9$ & 15,5 & 15,3 & 15,6 & 15,6 \\
\hline $6-6,9$ & 15,4 & 15,1 & 15,7 & 15,7 \\
\hline $7-7,9$ & 15,5 & 15,3 & 16,0 & 16,1 \\
\hline $8-8,9$ & 15,6 & 15,6 & 16,3 & 16,4 \\
\hline $9-9,9$ & 15,9 & 15,8 & 16,7 & 16,9 \\
\hline $10-10,9$ & 16,1 & 16,3 & 17,1 & 17,5 \\
\hline $11-11,9$ & 16,5 & 16,8 & 17,5 & 18,1 \\
\hline $12-12,9$ & 16,9 & 17,9 & 18,0 & 18,9 \\
\hline $13-13,9$ & 17,7 & 19,0 & 18,7 & 19,7 \\
\hline $14-14,9$ & 18,3 & 20,0 & 19,4 & 20,5 \\
\hline $15-15,9$ & 19,1 & 20,6 & 20,1 & 21,3 \\
\hline
\end{tabular}

a Instituto Brasileiro de Geografia e Estatística e Instituto Nacional de Alimentação e Nutrição, dados da Pesquisa Nacional sobre Saúde e Nutrição, 1989.

b Referência 21.

${ }^{c}$ As faixas de idade no estudo britânico foram relativas ao meio de cada intervalo, por exemplo: para o intervalo de 3 a 3,9 anos, o estudo britânico considerou a faixa de 3,5 anos.

TABELA 5. Valores médios do índice de massa corporal $\left(\mathrm{kg} / \mathrm{m}^{2}\right)$ por faixa etária das populações femininas brasileira, francesa e norte-americana

\begin{tabular}{ccccc}
\hline Faixa etária & Brasil $^{\mathrm{a}}$ & Estados Unidos $^{\mathrm{b}}$ & França $^{\mathrm{c}}$ & Estados Unidos $^{\mathrm{d}}$ \\
\hline $0-0,9$ & 16,8 & & 17,4 & \\
$1-1,9$ & 17,0 & & 16,2 & 16,1 \\
$2-2,9$ & 16,7 & & 16,0 & 15,6 \\
$3-3,9$ & 16,1 & & 15,6 & 15,5 \\
$4-4,9$ & 15,7 & & 15,3 & 15,6 \\
$5-5,9$ & 15,7 & $15,3 / 14,7^{e}$ & 15,3 & 15,7 \\
$6-6,9$ & & 15,4 & 16,1 \\
$7-7,9$ & 15,4 & & 15,6 & 16,4 \\
$8-8,9$ & 15,7 & & 16,0 & 17,5 \\
$9-9,9$ & 15,9 & & 16,5 & 17,8 \\
$10-10,9$ & 16,2 & & 17,0 & 18,9 \\
$11-11,9$ & 16,8 & 17,5 & 19,4 \\
$12-12,9$ & 17,5 & & 18,4 & 20,1 \\
$13-13,9$ & 18,5 & 19,3 & 21,1 \\
$14-14,9$ & 19,4 & 15,9 & 19,9 & 20,6 \\
$15-15,9$ & 20,4 & & 20,5 & 21,8 \\
$16-16,9$ & 20,8 & & 20,6 & 22,3 \\
$17-17,9$ & 21,5 & & 20,6 & 22,5 \\
$18-18,9$ & 21,7 & 20,8 & \\
$19-19,9$ & $21,0 / 20,1$ & 20,9 & \\
$20-20,9$ & 22,1 & & 21,0 & \\
$21-21,9$ & 22,1 & & & \\
$22-22,9$ & 21,8 & & & \\
$23-23,9$ & 22,2 & & & \\
$24-24,9$ & 22,4 & & & \\
\hline
\end{tabular}

a Instituto Brasileiro de Geografia e Estatística e Instituto Nacional de Alimentação e Nutrição, dados da Pesquisa Nacional sobre Saúde e Nutrição, 1989.

b Referência 30 .

c Referência 24.

d Referência 27.

e Valores obtidos através de dois modelos matemáticos diferentes. Idades não coincidem plenamente com os outros estudos. Por exemplo, nas três faixas etárias deste estudo, utilizaram-se os valores referentes às idades $5,5-6,5 ; 11,5-12,5$; e $17,5-18,5$.
Ainda em relação aos norteamericanos, os brasileiros apresentam valores médios de IMC semelhantes até os 6 anos e inferiores a partir dos 7 anos. Esta diferença chega a 2,1 UIMC para a faixa etária de 10 a 11 anos para o sexo feminino (tabela 5) e 2,7 UIMC para a faixa etária de 15 a 16 anos para o sexo masculino (tabela 6). Para a faixa de 1 a 19 anos (percentis e idades passíveis de comparação entre os dois estudos), as diferenças entre P 95 e P 5 são inferiores entre os americanos até 4 a 5 anos e, depois, são geralmente superiores; a maior diferença entre os percentis é de 5 UIMC (tabelas 7 e 8). Entre meninas, a maior diferença é verificada na faixa etária de 9 a 10 anos (27).

Em relação à França (24) os valores da população feminina brasileira são bastante semelhantes até os 16 anos e levemente superiores a partir desta idade (UIMC > 1). Já os dados da população masculina brasileira apresentam valores sistematicamente inferiores a partir dos 8 anos, com uma diferença entre 0,2 e 0,8 UIMC.

Um dado não disponível que seria interessante comparar é a idade a partir da qual o IMC se estabiliza, ou, pelo menos, passa a aumentar em um ritmo lento. Isto permitiria inferir se há diferenças, em termos populacionais, na idade em que o crescimento termina. No caso brasileiro, esta estabilização parece ocorrer em torno dos 20 anos, o que sugere ser esta a idade mínima em que o IMC pode ser utilizado, lançando-se mão dos limites de corte sugeridos na literatura $(13,31)$.

Comumente, em publicações que divulgam curvas de IMC produzidas em estudos semelhantes ao nosso, estimula-se o uso destas curvas como referência para fins de triagem (seleção, a partir de um ponto de corte, de indivíduos ou grupos que apresentam risco nutricional) e monitoramento ambulatorial (26), geralmente de pessoas obesas (12). Embora tentador dada a grande escassez de instrumentos de avaliação antropométrica de adolescentes e a facilidade de operacionalização do IMC, este tipo de aplicação de curvas de IMC deve ser reavaliado, por vários motivos. 
TABELA 6. Valores médios do índice de massa corporal $\left(\mathrm{kg} / \mathrm{m}^{2}\right)$ por faixa etária das populações masculinas brasileira, francesa e norte-americana

\begin{tabular}{ccccc}
\hline Faixa etária & Brasil $^{\mathrm{a}}$ & Estados Unidos $^{\mathrm{b}}$ & França $^{\mathrm{c}}$ & Estados Unidos $^{\mathrm{d}}$ \\
\hline $0-0,9$ & 17,2 & & 17,7 & \\
$1-1,9$ & 17,3 & & 16,6 & 16,3 \\
$2-2,9$ & 16,8 & & 16,1 & 15,9 \\
$3-3,9$ & 16,2 & & 15,8 & 15,8 \\
$4-4,9$ & 15,9 & & 15,6 & 15,6 \\
$5-5,9$ & 15,9 & 15,5 & 16,0 \\
$6-6,9$ & 15,6 & & 15,6 & 16,0 \\
$7-7,9$ & 15,7 & 15,9 & 16,6 \\
$8-8,9$ & 16,0 & 16,2 & 16,8 \\
$9-9,9$ & 16,2 & & 16,6 & 18,0 \\
$10-10,9$ & 16,5 & 16,9 & 18,7 \\
$11-11,9$ & $18,4 / 16,4$ & 17,4 & 18,8 \\
$12-12,9$ & 16,9 & 18,0 & 20,6 \\
$13-13,9$ & 17,3 & 18,7 & 20,8 \\
$14-14,9$ & 18,1 & & 19,6 & 22,1 \\
$15-15,9$ & 18,8 & & 20,0 & 21,7 \\
$16-16,9$ & 19,4 & & 20,7 & 22,7 \\
$17-17,9$ & 20,2 & 21,1 & \\
$18-18,9$ & 20,0 \\
$19-19,9$ & & 21,3 & \\
$20-20,9$ & $22,2 / 21,3$ & 21,8 & \\
$21-21,9$ & & 22,1 & \\
$22-22,9$ & & & \\
$23-23,9$ & 21,0 & & \\
$24-24,9$ & 21,4 & & & \\
\hline
\end{tabular}

a Instituto Brasileiro de Geografia e Estatística e Instituto Nacional de Alimentação e Nutrição, dados da Pesquisa Nacional sobre Saúde e Nutrição, 1989.

b Referência 30.

${ }^{c}$ Referência 24.

d Referência 27.

e Valores obtidos através de dois modelos matemáticos diferentes. Idades não coincidem plenamente com os outros estudos. Por exemplo, nas três faixas etárias deste estudo, utilizaram-se os valores referentes às idades 5,5-6,5; 11,5-12,5; e 17,5 - 18,5.

TABELA 7. Valores de percentis $(P)$ selecionados do índice de massa corporal $\left(\mathrm{kg} / \mathrm{m}^{2}\right)$ por faixa etária das populações femininas brasileira e norte-americana ${ }^{\mathrm{a}}$

\begin{tabular}{|c|c|c|c|c|c|c|c|}
\hline \multirow{2}{*}{$\begin{array}{l}\text { Faixa etária } \\
\text { (anos) }\end{array}$} & \multicolumn{3}{|c|}{ Estados Unidos $^{b}$} & \multicolumn{3}{|c|}{ Brasilc } & \multirow[b]{2}{*}{ Estados Unidos - Brasil $(a-b)$} \\
\hline & P 95 & P 5 & P $95-P 5(a)$ & P 95 & P 5 & $P 95-P 5(b)$ & \\
\hline $2-2,9$ & 18,5 & 14,1 & 4,4 & 19,2 & 14,3 & 4,9 & $-0,5$ \\
\hline $4-4,9$ & 18,0 & 13,7 & 4,3 & 18,0 & 13,6 & 4,5 & $-0,2$ \\
\hline $5-5,9$ & 19,6 & 13,5 & 6,1 & 18,3 & 13,2 & 5,0 & 1,1 \\
\hline $6-6,9$ & 19,3 & 13,6 & 5,7 & 18,2 & 13,3 & 4,9 & 0,8 \\
\hline $10-10,9$ & 24,1 & 13,7 & 10,4 & 21,0 & 13,8 & 7,2 & 3,2 \\
\hline $11-11,9$ & 26,2 & 14,9 & 11,3 & 22,8 & 14,1 & 8,6 & 2,7 \\
\hline $12-12,9$ & 26,3 & 14,9 & 11,4 & 23,4 & 14,7 & 8,7 & 2,7 \\
\hline $13-13,9$ & 28,5 & 15,2 & 13,3 & 24,3 & 15,4 & 8,9 & 4,4 \\
\hline $14-14,9$ & 28,8 & 16,4 & 12,4 & 26,0 & 16,1 & 9,9 & 2,5 \\
\hline $15-15,9$ & 26,6 & 17,1 & 9,5 & 26,0 & 17,2 & 8,9 & 0,6 \\
\hline
\end{tabular}

a Apresentadas só as faixas comuns aos dois estudos.

${ }^{\mathrm{b}}$ Referência 27.

c Instituto Brasileiro de Geografia e Estatística e Instituto Nacional de Alimentação e Nutrição, dados da Pesquisa Nacional sobre Saúde e Nutrição, 1989. 
TABELA 8. Valores de percentis $(\mathrm{P})$ selecionados do índice de massa corporal $\left(\mathrm{kg} / \mathrm{m}^{2}\right)$ por faixa etária das populações masculinas brasileira e norte-americana ${ }^{\mathrm{a}}$

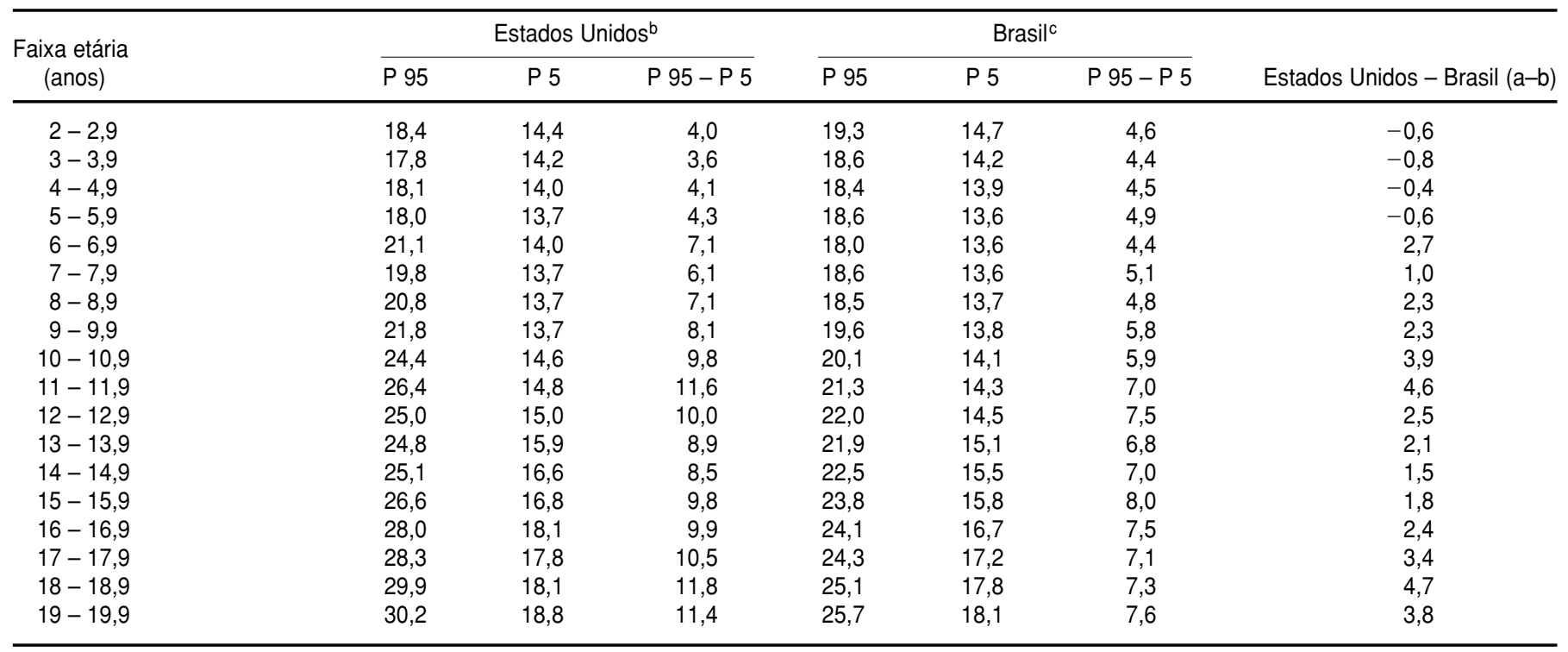

Apresentadas só as faixas comuns aos dois estudos.

${ }^{\mathrm{b}}$ Referência 27.

c Instituto Brasileiro de Geografia e Estatística e Instituto Nacional de Alimentação e Nutrição, dados da Pesquisa Nacional sobre Saúde e Nutrição, 1989.

Ao longo da adolescência, a composição corporal sofre intensas modificações e, também, diferenciações entre meninos e meninas. Assim, um mesmo valor de IMC, dependendo do gênero e do grau de maturação, terá diferentes significados em termos de composição corporal e, por conseguinte, de risco nutricional. Fica relativizada, portanto, a validade deste índice para os fins propostos (triagem e monitoramento ambulatorial). Para que a aplicação do IMC tenha validade, os instrumentos construídos devem levar em conta as modificações e diferenciações acima citadas, que não estão plenamente contempladas nas curvas construídas. Apesar do IMC ser semelhante entre os sexos durante a adolescência, com valores levemente superiores para a população feminina, os valores de dobras cutâneas variam, aumentando com a idade nas meninas e diminuindo nos meninos (13).

Além disso, há fatores limitantes para a adoção das curvas como referência: o primeiro diz respeito ao fato de a amostra estudada ser representativa de toda a população, incluindo, portanto, obesos e desnutridos. Portanto, não se pode afirmar que o perfil encontrado equivale ao desejável em termos de saúde, mas sim, que equivale ao perfil de uma dada população num dado momento. Logo, não parece prudente inferir níveis de risco nutricional a partir das curvas produzidas.

$\mathrm{O}$ segundo fator limitante refere-se à ausência de associação do IMC ao estagiamento de maturação sexual, principal variável condicionante do crescimento e desenvolvimento na adolescência. Como já comentamos, existem variações individuais em relação aos eventos pubertários. Por isto, o uso destas curvas para fins de triagem e monitoramento pode levar a diagnóstico equivocado. Por exemplo: duas meninas de 12 anos que apresentem o mesmo valor de IMC terão, a princípio, o mesmo diagnóstico antropométrico, quando deveriam ter diagnósticos e prognósticos diferentes, dependendo do seu estágio de maturação sexual.

$\mathrm{O}$ terceiro argumento leva em conta a discussão sobre os pontos de corte a serem adotados para a definição de risco nutricional em adolescentes. Esta tem sido dirigida para o diagnóstico de obesidade ou sobrepeso. Atualmente, tem sido cada vez mais corrente sugerir os percentis 85 e 95 ou 90 e 95 de curvas nacionais (norte-americanas, por exemplo) como pontos de corte para sobrepeso e obesidade $(12,28,29$, $32,33)$. Os principais questionamentos em relação a estas proposições referemse à pouca elucidação sobre a associação entre as faixas superiores a estes percentis e o risco de adoecer. Por outro lado, apontam para o fato de que, em populações que tendem à obesidade, os valores de IMC correspondentes a estes percentis podem ser mais altos que o desejável em termos de saúde. Na verdade, faltam informações sobre a característica composicional do aumento da massa corporal encontrada em várias populações e sua relação com a morbi-mortalidade, principalmente entre adolescentes.

Para desnutrição em crianças de até 10 anos e obesidade em adultos, a associação entre a antropometria e os agravos à saúde são bem estabelecidos. Para toda a população, os dados 
da PNSN evidenciam um quadro de ganho de estatura e massa corporal em relação aos dados obtidos durante o estudo nacional de despesas familiares (ENDEF) realizado na década de 70 (14). Apesar disso, os valores do percentil 95 do IMC da população adolescente brasileira são bastante inferiores aos da população norte-americana (figuras 3 e 4). É de se esperar que os valores para a população brasileira tenderão a aumentar e se aproximar dos valores norte-americanos em alguns anos, o que não parece desejável se o incremento de IMC representar deposição de gordura, como parece ocorrer na população americana $(34,35)$.

Em relação ao baixo peso, evento importante de ser acompanhado na realidade nacional, há discussão amadurecida somente para adultos com sugestão de limites de corte para tal fim (13). A mesma lógica de se usarem os extremos superiores da distribuição (P 85 e 95) poderia ser usada para se estabelecer o baixo peso, nesse caso com os valores extremos inferiores $(\mathrm{P}$ 15 ou 5). No caso do P 5, as curvas brasileiras e norte-americanas de IMC se assemelham, principalmente para a população feminina (figura 3). Fato aparentemente semelhante pode ser observado nos estudos britânico e francês $(24,25)$. Isto parece sugerir a possibilidade de haver limites de corte para baixo peso cujo uso poderia ser universal. Evidentemente, mais estudos são necessários, com incorporação do estagiamento maturacional e estabelecimento da relação entre o IMC e agravos à saúde, para que tais limites de corte possam ser sugeridos.

As curvas apresentadas de distribuição de IMC da população entre 0 e 25 anos trazem uma primeira contribuição para o conhecimento da distribuição deste índice na população brasileira. Deve-se garantir, em estudos futuros com adolescentes, a associação da maturação sexual às medidas antropométricas. O perfil encontrado expressa diferenças esperadas na maturação entre a população masculina e feminina e assemelha-se, em linhas gerais, aos de estudos realizados na França e nos Estados Unidos.
FIGURA 3. Percentis (P) selecionados do índice de massa corporal $\left(\mathrm{kg} / \mathrm{m}^{2}\right)$ das populações femininas brasileira ${ }^{a}$ norte-americana ${ }^{b}$ em função da idade

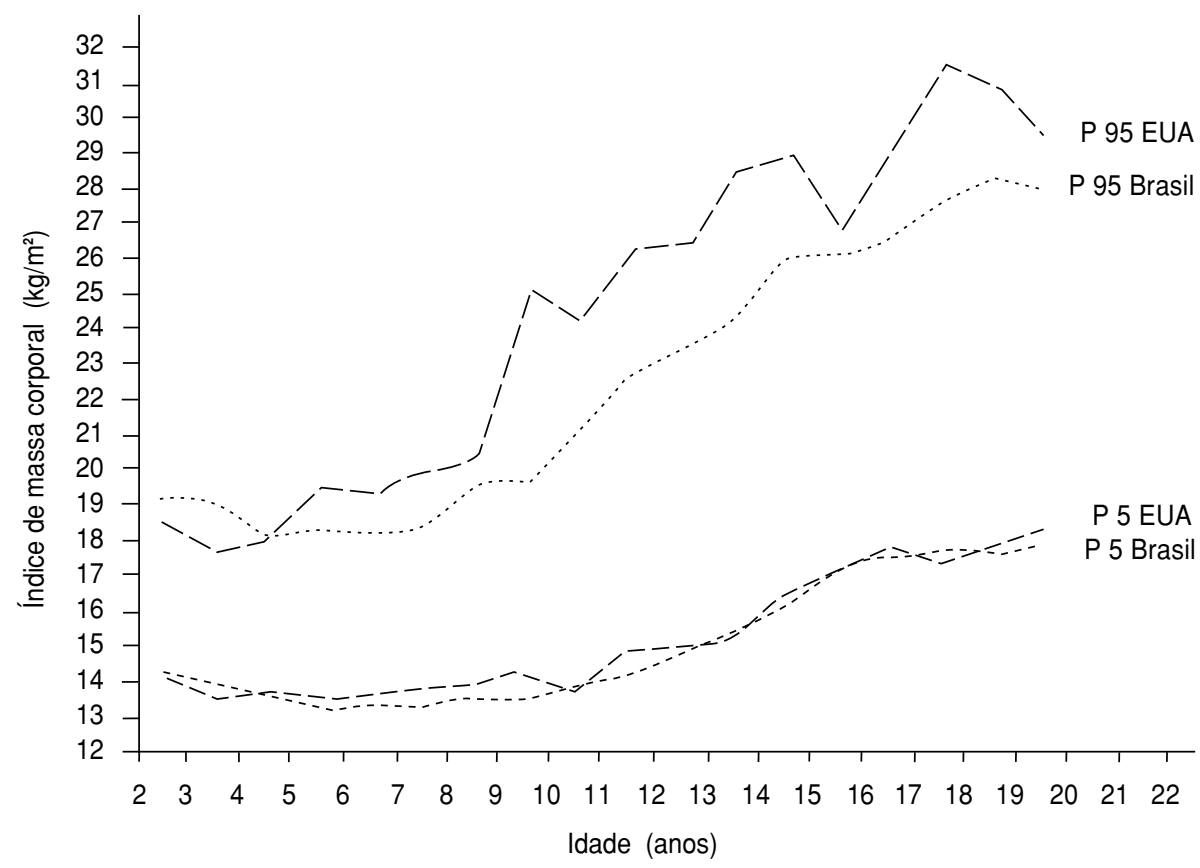

a Instituto Brasileiro de Geografia e Estatística e Instituto Nacional de Alimentação e Nutrição, dados da Pesquisa Nacional sobre Saúde e Nutrição, 1989.

${ }^{b}$ Referência 27.

FIGURA 4. Percentis (P) selecionados do índice de massa corporal $\left(\mathrm{kg} / \mathrm{m}^{2}\right)$ das populações masculinas brasileira ${ }^{\mathrm{a}}$ e norte-americana ${ }^{\mathrm{b}}$ em função da idade

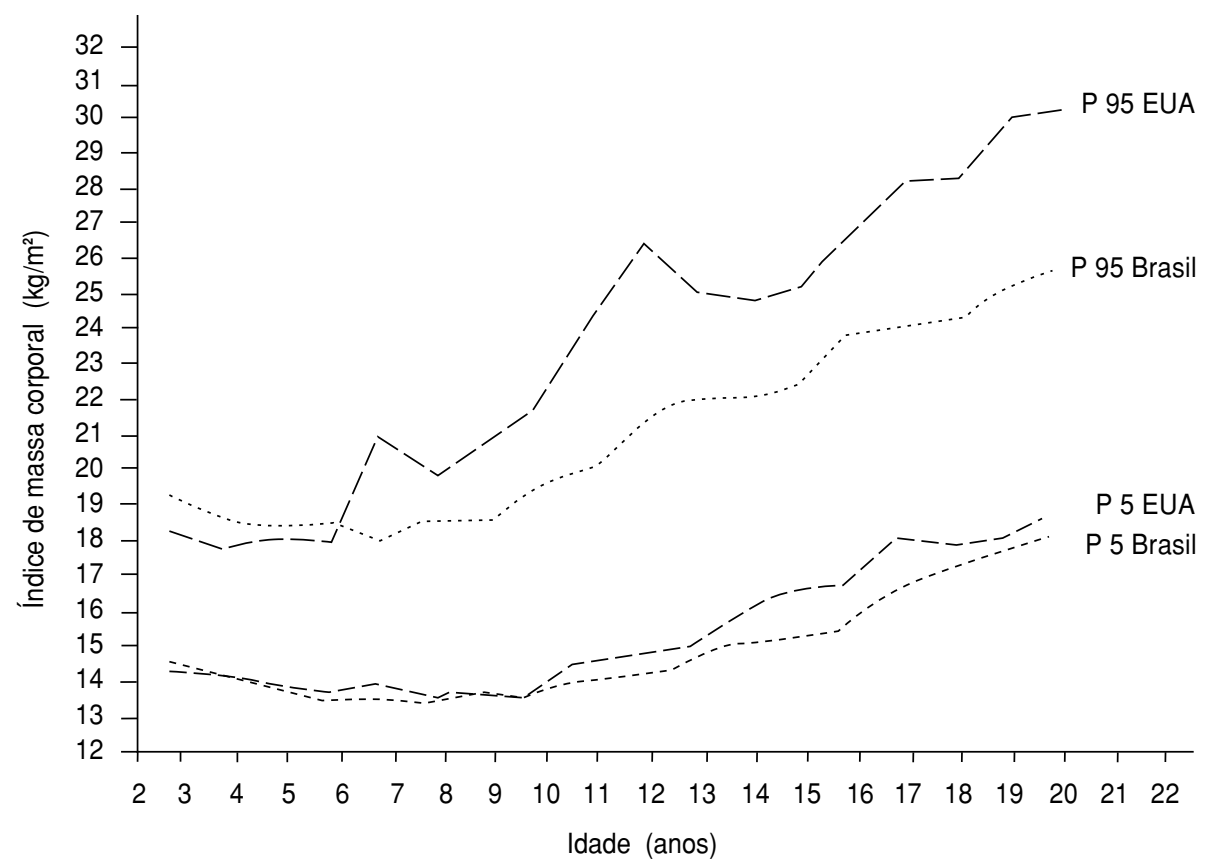

a Instituto Brasileiro de Geografia e Estatística e Instituto Nacional de Alimentação e Nutrição, dados da Pesquisa Nacional sobre Saúde e Nutrição, 1989.

${ }^{\mathrm{b}}$ Referência 27. 


\section{REFERÊNCIAS}

1. Suárez A, Arguilles JM. Nutritional evaluation of adolescents: usefulness of anthropometric indicators in the diagnosis of obesity. Acta Paediatr Hung 1986;27:303-310.

2. Heald EP. Adolescent nutrition. Med Clin North Am 1975;59(6):1329-1336.

3. Duarte MFS. Maturação física: uma revisão da literatura, com especial atenção à criança brasileira. Cad Saude Publica 1993;9(supl 1):71-84.

4. Malina RM. Adolescent growth and maturation: selected aspects of current research. Yearb Phys Anthropol 1978;21:63.

5. Tanner JM. Growth at adolescence. Oxford: Blackwell Scientific Publications; 1962.

6. Saito MI. Desnutrição. Em: Coates V, Françoso LA, Beznos GW, eds. Medicina do adolescente. São Paulo: Sarvier; 1993:59-65.

7. Habicht JP, Martorell R, Yarbrough C, Malina RM, Klein RE. Height and weight standards for preschool children: How relevant are ethnic differences in growth potential? Lancet 1974;1(858):611-614.

8. Waterlow JC, Buzina R, Keller W, Lane JM, Nichaman NZ, Tanner JM. Presentation and use of height and weight data for comparing the nutritional status of groups of children under the age of 10 years. Bull World Health Organ 1977;55(4):489-498.

9. World Health Organization. Use and interpretation of anthropometric indicators of nutritional status. Bull World Health Organ 1986;64(6):929-941.

10. Roche AF, Siervogel FM, Chumlea WC, Webb P. Grading body fatness from limited anthropometric data. Am J Clin Nutr 1981;34: 2831-2838.

11. Veiga GV, Sampei MA, Sawaya AL, Sigulem DM. Adaptação do critério antropométrico para avaliação do estado nutricional de adolescentes em dois níveis socioeconômicos. J Pediatr (Rio J) 1992;68:26-33.

12. Himes JH, Dietz WH. Guidelines for overweight in adolescent preventive services: recommendations from an expert committee. Am J Clin Nutr 1994;59(2):307-316.

13. Anjos LA. Índice de massa corporal $\left(\mathrm{kg} / \mathrm{m}^{2}\right)$ como indicador do estado nutricional de adultos: uma revisão da literatura. Rev Saude Publica 1992;26:431-436.

14. Brasil, Ministério da Saúde, Instituto Nacional de Alimentação e Nutrição (INAN). Perfil de crescimento da população brasileira de 0 a 25 anos: Pesquisa Nacional sobre Saúde e Nutrição. Brasília: INAN; 1990.

15. Satyanarayana K, Krishna TP, Rao BSN Effect of early childhood undernutrition and child labour on growth and nutritional status of rural Indian boys around Hydebarad. Hum Nutr Clin Nutr 1986;40(2): 131-139.

16. Duarte MFS. Maturação física: uma revisão da literatura, com especial atenção à criança brasileira. Cad Saude Publica 1993;9(supl 1):71-84.

17. Forbes GB. Human body composition: Growth, aging, nutrition and activity. New York: Springer-Verlag; 1987.

18. Fletcher PR [documento mimeografado]. Pesquisa nacional sobre saúde e nutrição — plano de amostragem. Brasília: OIT/PNUD/IPLAN/ IPEA; 1987.

19. Fletcher PR [documento mimeografado]. Pesquisa nacional sobre saúde e nutrição: a seleção dos elementos da amostra na segunda etapa de amostragem. Brasília: OIT/PNUD/IPLAN/ IPEA; 1988

20. Waaler HT. Hazard of obesity: the Norwegian experience. Acta Med Scand Suppl 1988;723: 17-21.

21. SAS/STAT 6.03 [programa de computador]. Gary, North Carolina: SAS Institute Inc; 1988

22. Heald EP. Adolescent nutrition. Med Clin North Am 1975;59:1329-1336.

23. Marino DD, King JC. Nutritional concerns during adolescence. Pediatr Clin North Am 1980;27:125-139.

24. Rolland-Cachera MF, Cole TJ, Sempé M, Tichet J, Rossignol C, Charraud A. Body mass index variations: centiles from birth to 87 years. Eur J Clin Nutr 1991;45:13-21.

25. White EM, Wilson AC, Greene SA, McCowan C, Thomas GE, Cairns AY, Ricketts IW. Body mass index centile charts to assess fatness of British children. Arch Dis Child 1995;72:38-41.
26. Cole TJ, Freeman JV, Preece MA. Body mass index reference curves for the UK, 1990. Arch Dis Child 1995;73:25-29.

27. National Center for Health Statistics (NCHS). Anthropometric reference data and prevalence of overweight. DHHS Publication No (PHS) 87$1688 ; 1987$.

28. Hammer LD, Kraemer HC, Darrel MW, Ritter PL, Dornbusch SM. Standardized percentile curves of body-mass index for children and adolescents. Am J Dis Child 1991;145:259-263.

29. Must A, Dallal GE, Dietz WH. Reference data for obesity: 85th and 95th percentiles of body mass index (wt/ht2) and triceps skinfold thickness. Am J Clin Nutr 1991;53(4):839-846.

30. Siervogel RM, Roche AF, Guo S, Mukherjee D, Chumlea WC. Patterns of change in weight/ stature from 2 to 18 years: findings from longterm serial data for children in the Fels longitudinal growth study. Int J Obes 1991;15: 479-485.

31. Anjos LA. O índice de massa corporal só deve ser usado em indivíduos com mais de 20 anos de idade. Em: Centro de Estudos do Laboratório de Aptidão Física de São Caetano do Sul (CELAFISCS). Anais do XIX Simpósio Internacional de Ciências do Esporte. São Caetano do Sul, SP: CELAFISCS; 1994:194.

32. Maffeis C, Schutz Y, Piccoli R, Gonfiantini E, Pinelli L. Prevalence of obesity in children in north-east Italy. Int J Obes 1993;17: 287-294.

33. Malina RM, Zavaleta AN, Little BB. Estimated overweight and obesity in Mexican-American school children. Int J Obes 1986;10:483-491.

34. Gortmaker SL, Dietz WH, Sobol AM, Wheler CA. Increasing pediatric obesity in the United States. Am J Dis Child 1987;141:535-540.

35. Lohman TG. Advances in body composition assessment. Champaign, Illinois: Human Kinetics; 1992. em versão revisada em 6 de setembro de 1996.
ABSTRACT

\section{Distribution of body mass index in the Brazilian population under 25 years of age}

This paper presents the percentage distribution of the body mass index of the Brazilian population from birth to age 25 , based on a national survey conducted in 1989 . Survey data show that body mass index decreases from birth until around 6 years of age, reaching a plateau at 8 years and progressively increasing until the age of 19 or 20 years for females and 20 or 21 for males. The survey also revealed that after the age of 12 females present a greater body mass index than males, as well as a larger range of percentile values (from 3 to 97). A comparison with data from other countries showed that the body mass index profile in Brazil is similar to that observed in France, Great Britain, and the United States. Before the age of 6, Brazilian youngsters have a mean body mass index that resembles that of North American children, and a lower one thereafter. A comparison between the median body mass index of Brazilian and British youngsters revealed consistently lower values among Brazilian females. When compared to that of France, Brazil's male population has a systematically lower body mass index after the age of 8 . It is suggested that the data presented in our study be used only for comparing groups of individuals and studies, and not for screening or clinical monitoring, due to the great variability in growth patterns during adolescence. 